\title{
¿Quién cuida de quién? La (des)responsabilización de las condiciones, reproducción y finitud de la vida
}

\section{Candela Rocío Heredia}

Universidad Nacional de San Martín (UNSAM)

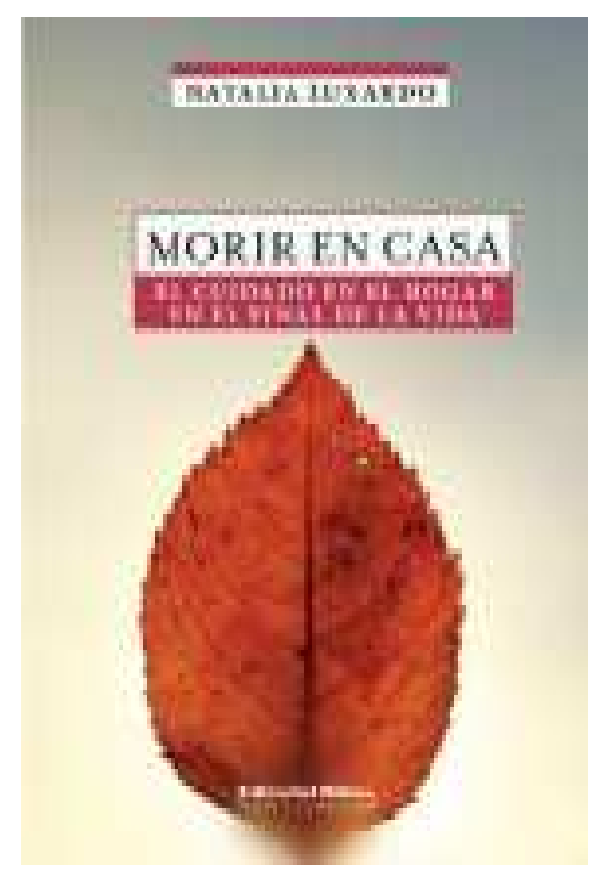

RESEÑA: ¿Quién cuida de quién? La (des)responsabilización de las condiciones, reproducción y finitud de la vida

LUXARDO, Natalia. Morir en casa. El cuidado en el hogar en el final de la vida. Buenos Aires: Biblos, 2011.

BOOK REVIEW: Who Cares for Whom? Not Taking Responsibility for Conditions, Reproduction and the Limits of Life

LUXARDO, Natalia. Morir en casa. El cuidado en el hogar en el final de la vida. Buenos Aires: Biblos, 2011. 
La intensificación del debate en torno a las políticas sociales de salud trae a discusión el papel de los/as profesionales, las familias, las instituciones públicas y entidades privadas en el área del cuidar de las poblaciones. El cuidado, en tanto acción social y política que garantiza la reproducción de las sociedades, amerita una profundización teórico-analítica en sus postulados básicos, al tiempo que requiere de sólidas demandas profesionales y ciudadanas alineadas en una perspectiva emancipadora y transformadora de las relaciones desiguales de poder-saber. La importancia e implicancias del cuidado refieren a la polisemia de la noción en auge, a las necesidades de los sujetos concretos y al impacto de las políticas sociales de salud en las condiciones de vida. En efecto, frente a la actual sociedad de clase y pluricultural, caracterizada demográficamente por un envejecimiento poblacional y, epidemiológicamente, por un aumento de enfermedades de larga duración, se abre el debate de cómo actuar en sus principales problemáticas posicionándose desde un proyecto éticopolítico. Morir en casa es un aporte a este debate de la dimensión política de los cuidados, promoviendo las preguntas referidas a la configuración de la responsabilización de las condiciones, reproducción y finitud de la vida. El libro aborda específicamente los cuidados de personas con enfermedades terminales, colocando el énfasis en tres elementos: a) el hogar como espacio de provisión de atención-cuidados; b) la persona con enfermedad; y c) la persona encargada de realizar el cuidado.

La obra de Natalia Luxardo coloca bajo la mira las tendencias mundiales de incremento de la esperanza de vida, disminución de la tasa de natalidad, reducción de la familia extensa, migraciones de la población económicamente activa y prevalencia de enfermedades crónico-degenerativas sobre las infecto-contagiosas, las cuales ponen en evidencia la creciente presencia de personas dependientes y de demanda de cuidados, que se ven enfrentadas a la menor disponibilidad del núcleo familiar de ejercerlos. La necesidad de dispositivos capaces de atender a estas problemáticas invita a alejarse de un tratamiento profuso de la cuestión relativa a los cuidados, entendiendo a los mismos como una acción política engarzada con una amalgama de valores morales, éticos y estéticos. Hablar de formas de cuidados implica hablar de formas de vida y es, eminentemente, un objeto de disputa en la actual arena política, en tanto acarrea a los responsables y dispositivos operantes más idóneos para su ejecución: ¿Serán las familias las encargadas de tal labor? ¿El Estado a través de sus políticas? ¿Quién cuida de quién? ¿Bajo qué premisas y directrices lo hace? El libro de Natalia Luxardo reaviva estas preguntas atinentes a los cuidados desde una ubicación estratégica: puntualizando lo que acontece en los trayectos de fin de vida, con una descripción densa de las vivencias de los sujetos involucrados.

En un primer momento, la autora nos introduce a la temática trayendo a colación las definiciones de muerte y actitudes hacia la muerte, preguntándose sobre el surgimiento del concepto de buena muerte o muerte digna. Esta introducción conceptual y de caracterización de sus dilemas inescindibles, se muestran como elemento angular en los intentos prácticos, familiares e institucionales, de garantizar un tránsito de fin de vida en ese orden. ¿Cómo morir con dignidad? ¿A qué personas y entidades involucra? La autora liga las dimensiones de tales interrogantes y las construye como foco de indagación.

A lo largo del libro, describe detalladamente las particularidades del mundo de la persona con enfermedad y el mundo del cuidador/a(s). Con un nivel descriptivo y de revisión de literatura asombrosamente meticuloso, introduce a los/as lectores/as a tales mundos colocando como espacio observacional, el hogar. Sin embargo, antes de sumergirnos en dichos mundos, la autora realiza una contextualización de las actuales formas de tránsito de final de vida. De esta manera, señala que, a pesar de erigirse el hogar como el lugar ideal de cuidados-muerte para la mayoría de las personas, en los países occidentales el $70 \%$ fallece en hospitales. Estos decesos son problemáticos para los gobiernos por el alto costo de hospitalización. Ante esta advertencia financiera para los gobiernos, la autora señala intervenciones de políticas que pueden sustentar la promoción de la muerte en el hogar. No obstante, la aplicación de tales intervenciones (como pueden ser los cuidados paliativos o internaciones domiciliarias) pueden redundar en una delegación de responsabilidades, encargando a las familias la atención de las personas con enfermedades. Así, la familiarización de la atención de la salud puede ser un factor en este traslado de dispositivos sanitarios, motivo por el cual, nos exhorta a una lectura compleja de estas medidas que intentan un cambio del lugar de cuidado en fin de vida y del lugar de muerte.

La atención que se realice en el hogar tiene un abanico de posibilidades. La médica Vilma Tripodoro y la trabajadora social Elena D'Urbano realizan su aporte en este sentido, escribiendo un único capítulo ${ }^{1}$ sobre la atención que realizan en domicilio desde la medicina paliativa. Desarrollan un análisis del encuadre legal vigente en Argentina en relación a los cuidados paliativos domiciliarios, concluyendo en la falta de una respuesta unificada, estructurada y concreta desde el sistema público sanitario. Asimismo, marcan fuertemente los beneficios que conlleva la inversión en medicina paliativa domiciliaria, tanto para disminuir la utilización de camas de agudos como para bienestar de las familias, dado que "la medicina paliativa no sólo se ocupa de la atención del paciente, sino que se hace extensiva al cuidado de la familia [...]" (p. 114).

Las familias que ejercen los cuidados informales en el hogar, despliegan una serie de estrategias para la atención a la persona enferma. La organización diaria de estos cuidados se realiza dentro del ámbito doméstico, 
ámbito donde se funden los dos mundos: del enfermo y del cuidador. "El mundo del enfermo", como lo llama la autora, es un mundo cargado de vivencias heterogéneas; vaivenes en los procesos de salud-enfermedad; emociones y sentires complejos y dolorosos; tratamientos terapéuticos, paliativos, alternativos, tradicionales, convencionales, ficticios; corporalidad mutable; identidad deteriorada, contingente, híbrida, dependiente; biografía resignificada; cambios abruptos y constantes; creencias religiosas y/o espirituales en la búsqueda de sostén simbólico; afectividad exteriorizada y eclipsada; tiempos de incertidumbre; pronósticos de muerte y anhelos de sanación. El mundo del enfermo es complejo, estructurado por momentos, caótico en otros, y deslumbrante siempre, en tanto fenómeno que permite una aproximación a la generalidad de la vida social.

"El mundo del cuidador", aquel que es inexorable al mundo del enfermo, merece en la obra un capítulo aparte. La denominación "cuidador" refiere a los intentos por conceptualizar a estos/as encargados/as de llevar adelante la labor de cuidar. Esta denominación puede adscribir connotaciones no amenas para las personas calificadas como tal, ya que, como se cita a Ellis-Hill, "cuando una persona es definida como cuidador/a, sólo existe en relación con la persona cuidada. No existe como una persona en su propio derecho, con sus propias necesidades" (p. 112). Con precaución ante esta posibilidad de caer en una ocultación de las necesidades particulares y derechos de las personas encargadas del cuidado, Luxardo describe el mundo del cuidador trayendo sus propias voces y detallando tanto los matices comunes como las singularidades de los/as cuidadores/ as. Cuidadores preparados y organizados en la previsión óptima del cuidado, con apoyo de familiares, amigos, compañeros y/o vecinos; cuidadores al borde de la

... Morir en casa es una

\section{producción cuya riqueza se}

encuentra en la estratégica

ubicación a partir de la cual

cuestionar los senderos y

derroteros de la

responsabilización de las

condiciones, reproducción y

finitud de la vida. claudicación; cuidadores vulnerables conformando una díada frágil con la persona cuidada; cuidadores con inquietudes respecto del cuidado; con formas y contenidos de la comunicación específicos; con soporte social; con apoyo emocional; demandantes de mayor intervención médica; rechazantes de ayuda de un equipo de salud; con trámites y gestiones sanitarias que realizar; variados son los perfiles de personas que, como pueden, intentan cuidar.

Ya concluyendo, la autora señala que "la muerte en casa sin estrategias de apoyo óptimas y sustentables en el largo plazo, brindadas y garantizadas por el sistema formal a través de políticas de asistencia concretas, puede transformarse en un proceso dañino para la salud física y psíquica del cuidador" (p. 243), evidenciando, entonces, el carácter fundamental de políticas sociales de salud que no trasladen la responsabilidad del cuidado a las familias, que no carguen a ellas además, el peso de haber sido responsables de una "mala muerte". La finalidad de la vida de los sujetos que nos muestra la autora, posibilita así, contemplar todos estos juegos, de determinaciones y mediaciones de la vida, de una responsabilización en disputa en los ámbitos institucionales y socio-culturales de la vida social.

En este sentido, el mundo del enfermo y del cuidador en el hogar, son mundos que observándolos, nos abre las puertas al mundo de la organización social de la vida. La finitud humana, que parecería descubrirse en el momento de fin de vida de una persona, muestra su naturaleza histórica, la cual permite ver los encadenamientos de condiciones de la existencia, que se reprodujeron o no, en esa vida. Pues, querer que la vida finalice de la forma en que se quiere haber vivido, hacen de la finitud (conclusión de la existencia) una ventana para la inspección de las trayectorias de vida concretas de los sujetos.

En resumen, Morir en casa es una producción cuya riqueza se encuentra en la estratégica ubicación a partir de la cual cuestionar los senderos y derroteros de la responsabilización de las condiciones, reproducción y finitud de la vida. Desde las vivencias de los sujetos en final de vida y sus cuidadores/as, nos muestra las implicancias de tales caminos tomados. De esta forma, el libro contribuye a visibilizar las problemáticas, dando una descripción y análisis crítico de las respuestas otorgadas. Por otro lado, en lo que respecta al aspecto profesional, permite conocer las particularidades en detalle de un campo ocupacional sanitario (los cuidados en domicilio y paliativos), pero aporta, sobretodo, a construir una mediación necesaria para la planificación-acción en instituciones, consolidando la autonomía profesional. El libro constituye una contribución de importancia a las formas de investigación e intervención en la nueva coyuntura demográfica y epidemiológica, que desafía a los agentes encargados del diseño, gestión y ejecución de políticas públicas en el camino de la protección social de los sujetos en su integralidad. 


\section{Nota}

1 El libro es enteramente de autoría de Luxardo, a excepción de un capítulo (el número 4) y un anexo (el número 2) que es escrito por Tripodoro y D’Urbano. Los restantes capítulos (en total son seis) y el primer anexo, son de la autora.

\section{Candela Rocío Heredia}

candelarheredia@gmail.com

Maestranda en Antropología Social por el Instituto de Desarrollo Económico y Social (IDES) e Instituto de Altos Estudios Sociales (IDAES-UNSAM)

\section{Universidad Nacional de San Martín (UNSAM/Argentina)}

Campus Miguelete, 25 de Mayo y Francia

San Martín

Provincia de Buenos Aires - Argentina

C.P.: 1650 